\title{
Biological functions of DEAD/DEAH-box RNA helicases in health and disease
}

\author{
On 15-16 November 2021, the National Institute of Allergy and Infectious Diseases (NIAID), the National Cancer \\ Institute ( $\mathrm{NCl}$ ) and the National Heart, Lung, and Blood Institute (NHLBI) hosted a virtual workshop on DEAD/ \\ DEAH-box RNA helicases in health and disease. The goal of the workshop was to review current advances, and \\ identify knowledge gaps and future research to improve our understanding of the function of RNA helicases, and \\ leverage these molecules as molecular targets with translational potential.
}

$\mathrm{D}$ EAD/DEAH-box RNA helicases (RHs) are highly conserved RNA-binding proteins with ATPase activity. They are crucial for RNA metabolism ${ }^{1}$, and belong to the $\mathrm{RH}$ superfamily 2 (SF2) that is characterized by nine conserved motifs. These motifs mediate ATP binding and hydrolysis, RNA binding and ATP-dependent intramolecular RNA remodeling. These RHs are defined by their Asp-Glu-Ala-Asp (DEAD) or Asp-Glu-Ala-His (DEAH) amino acid sequences ${ }^{2}$. Although some of these RHs unwind duplex RNA, others can unwind double-stranded DNA (dsDNA) and RNA-DNA duplexes, whereas others exhibit little to no unwinding activity. The human genome encodes 37 DEAD-box and 16 DEAH-box RHs that exhibit diverse biological functions, including the regulation of embryonic development, cell proliferation, hematopoiesis, metabolism, innate immunity and immune programming, cancer pathogenesis, inflammation, and autoimmune diseases (Fig. 1 and Table 1). Several RHs sense cytosolic non-self viral RNAs and initiate antiviral innate immune signaling ${ }^{3}$. Moreover, RHs have crucial roles in genome integrity; ${ }^{4}$ mutations in $\mathrm{RHs}$ are linked to cancers, autoimmunity and other diseases ${ }^{5}$. Thus, DEAD/DEAH-box RHs are attractive therapeutic targets ${ }^{3,6}$. Despite the progress in this field, key knowledge gaps remain, including cofactors of $\mathrm{RH}$ regulation; the nature of their RNA substrate; post-translational modifications that regulate RH function; and the role of RHs in signaling cascades of biological processes. This National Institutes of Health workshop focused on recent advances in understanding the functions of DEAD/ DEAH-box RHs.

\section{Biology and basic functions of DEAD/ DEAH-box RHs}

Rick Russell (University of Texas) kicked off the meeting with an overview of the structure, activities and functional roles of
RHs in mediating RNA structural transitions and maintaining RNA in unfolded or underfolded states. Examples of the role of RHs in regulating gene expression include how deacetylation of the helicase DDX3X (also known as DDX3) by HDAC6 regulates the formation of stress granules through liquid-liquid protein phase separation (LLPS), presented by Patrick Matthias (Friedrich Miescher Institute for Biomedical Research). Stress granules are membrane-less organelles that are the sites of stalled mRNA translation, and are involved in the stress response. Stéphane Richard (McGill University) discussed the role of DDX5 in resolving R-loops, DNARNA hybrids and associated non-template single-stranded DNA. DDX5 methylation by protein arginine methyltransferase 5 within its RGG/RG motif enables interaction with $\mathrm{XRN} 2$ ribonuclease, leading to the resolution of R-loops and ensuring DNA repair. Kevin Raney (University of Arkansas) described the binding of yeast Ded1 (human homolog DDX3X) to G-quadruplex (G4) DNA, leaking from the nucleus or mitochondria, initiating LLPS and stress granule formation. Timothy Weil (University of Cambridge) discussed ribonucleoprotein processing (P)-bodies in mRNA storage in Drosophila oocytes, showing that Me31B (DDX6 in humans) in P-body condensates stores bicoid mRNA, and releases it for translation upon egg activation. Finally, Danzhou Yang (Purdue University) discussed how DDX5 unfolds the G4 structure at the MYC promoter, and regulates $M Y C$ transcription. This session described specific function of distinct DEAD/DEAH-box helicases in resolving complex RNA-DNA structures to facilitate genome integrity and regulate gene expression.

\section{Organ-specific infection and cancer Deregulated expression of various RHs has a role in cancer pathogenesis, as well as in infection and cellular differentiation. Specifically, Elizabeth Tran (Purdue University) reported the linkage of DDX5}

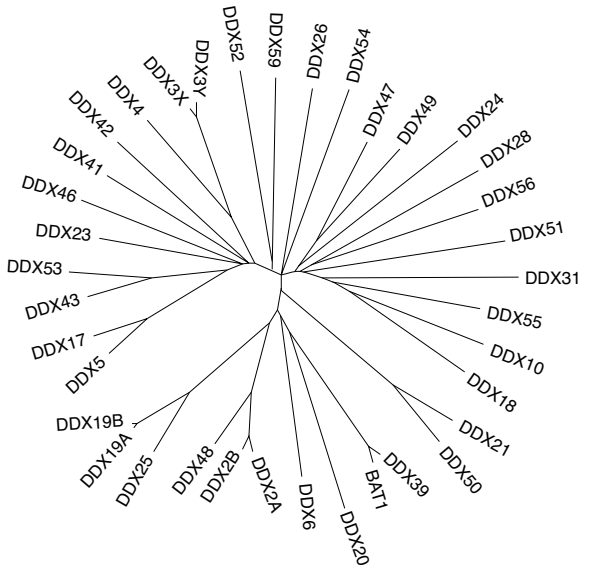

Fig. 1 | Phylogeny of human DEAD/DEAH-box RHs. Phylogenetic analysis performed using the Poisson model of amino acid substitution with the neighbor-joining method and scaled with the branch lengths representing the evolutionary distances. The evolutionary distances to infer the phylogenetic tree were in the unit of the number of substituting amino acids per site.

with small cell lung cancer (SCLC), and reported that depletion of DDX5 reduced the growth of a chemoresistant SCLC cell line by altering mitochondrial function. By contrast, in hepatocytes, DDX5 forms an epigenetic silencing complex with IFI16, encoded by an interferon-stimulated innate immunity gene, via an RNA-dependent mechanism, which suggests that loss of this complex enables hepatitis B virus transcription and hepatocellular carcinoma progression $^{7}$, as presented by Ourania Andrisani (Purdue University). William Ricke (University of Wisconsin) described how DDX3X promotes androgen receptor $(\mathrm{AR})^{\text {neg/low }}$ castration-resistant prostate cancer (CRPC) by binding to AR mRNA and suppressing $A R$ translation, thereby identifying DDX3X as a promising therapeutic target for the treatment of CRPC. Stephen Floor (University of California, San Francisco) discussed 
Table 1 | RHs and their functions featured in the workshop

\begin{tabular}{|c|c|c|c|}
\hline \multicolumn{3}{|c|}{ DEAD/DEAH-box RHs } & \multirow{2}{*}{$\begin{array}{l}\text { Function } \\
\text { - Supports gastrulation and central nervous system development }\end{array}$} \\
\hline SF1 & \multicolumn{2}{|c|}{ Mov10 } & \\
\hline \multirow[t]{8}{*}{ SF2 } & \multicolumn{2}{|c|}{ DDX3X } & $\begin{array}{l}\text { - Suppresses AR translation in } \mathrm{AR}^{\text {low/neg }} \text { castration-resistant prostate cancer } \\
\text { - Inhibition induces cell death and suppresses latent HIV infection } \\
\text { - Involved in LLPS and stress granule formation } \\
\text { - A tumor suppressor that generates medulloblastoma subtypes } \\
\text { - Knockdown triggers anti-tumor immunity via production of IFN or STAT }\end{array}$ \\
\hline & \multicolumn{2}{|l|}{ DDX5 } & $\begin{array}{l}\text { - Resolves DNA-RNA hybrids (R-loops) } \\
\text { - Promotes MYC transcription by unfolding a G-quadruplex of the MYC } \\
\text { promoter } \\
\text { - Supports cellular respiration in small cell lung cancer cell lines } \\
\text { - Forms an RNA-driven silencing epigenetic complex with innate nuclear } \\
\text { receptor IFI16 in hepatocytes }\end{array}$ \\
\hline & \multicolumn{2}{|l|}{ DED1 } & $\begin{array}{l}\text { - Binds and unfolds RNA and DNA quadruplexes } \\
\text { - Coordinates with elF4 to stimulate preinitiation complex formation }\end{array}$ \\
\hline & \multicolumn{2}{|c|}{ Me31B } & - Forms viscous P body condensates with bicoid mRNA in Drosophila \\
\hline & \multicolumn{2}{|c|}{ DDX41 } & $\begin{array}{l}\text { - Senses retroviruses, supports innate immunity and HSPC differentiation; } \\
\text { limits R-loops } \\
\text { - Mutations cause human myeloid neoplasms and myelodysplastic } \\
\text { syndromes }\end{array}$ \\
\hline & \multicolumn{2}{|c|}{ Antiviral Dicer } & $\begin{array}{l}\text { - Enriched in stem cells within adult tissues; protects adult stem cells from } \\
\text { RNA viruses via canonical antiviral RNAi response }\end{array}$ \\
\hline & \multicolumn{2}{|c|}{ DDX56 } & - Maintains stemness in normal and cancer stem cells \\
\hline & RLRs & $\begin{array}{l}\text { DDX58 } \\
(\mathrm{RIG}-1) \\
\text { DHX58 } \\
\text { (LGP2) } \\
\text { IFIH1 } \\
\text { (MDA5) }\end{array}$ & $\begin{array}{l}\text { - Pathogen recognition } \\
\text { - Initiator of innate immunity } \\
\text { - Immune system activation and programming; RLR agonists exhibit } \\
\text { anti-viral and anti-tumor effects }\end{array}$ \\
\hline
\end{tabular}

DHX15 - Immune system activation; senses rotavirus infection in intestinal epithelia; shown to sense RNA viruses in specific cell types

Functions of RHs that featured in the 'Biological Functions of DEAD/DEAH-box RNA Helicases in Health and Disease' workshop.

how DDX3X controlled the translation of mRNAs enriched in RNA structures, which resulted in altered transcriptome output in both cancer and developmental disorders. Furthermore, Richard Gilbertson (University of Cambridge) described DDX3X mutations in medulloblastoma, which indicates that DDX $3 \mathrm{X}$ is a tumor suppressor that restricts the number of cell lineages that can generate medulloblastoma subtypes $^{5}$. Susan Ross (University of Illinois) discussed the role of DDX41 in recognizing retroviral RNA-DNA products of reverse transcription, and acting as an upstream regulator of innate immune signaling. DDX41 is also crucial for the differentiation of hematopoietic stem and progenitor cells (HSPCs). Loss-of-function $d d x 41$ mutations lead to loss of myeloid cells and susceptibility to myelodysplastic syndromes (MDS), as discussed by Teresa Bowman (Albert Einstein College of Medicine). Zebrafish $d d x 41$ mutants have aberrant HSPC expansion, R-loop imbalances and increased expression of inflammatory genes, which triggers innate immune activation and HSPC expansion ${ }^{8}$. Finally, Daniel Starczynowski (Cincinnati Children's Hospital) discussed mutations linked to MDS in mice, showing that monoallelic mutations in $D d x 41$ result in features of MDS in an age-dependent manner. Overall, this session showed that RHs mediate cell context-dependent, specialized functions, linked to interactions between cofactors and RNA substrates.

\section{Development and cell regulation}

Several RHs are required for eukaryotic translation and are important for maintaining the integrity of protein synthesis. Alan Hinnebusch (National Institute of Child Health and Human Development) discussed the mechanism of translation initiation in yeast by eukaryotic initiation factor eIF4A and Ded1. Ded1 and eIF4A stimulate translation by resolving RNA structures that impede the attachment of the preinitiation complex or 5-nontranslated scanning. Enzo Poirier
(Crick Institute) discussed the identification of a new mammalian antiviral Dicer, an alternatively spliced Dicer mRNA without a Hel2i domain. Antiviral Dicer is highly efficient at processing dsRNA and protects adult stem cells from RNA viruses by degrading viral dsRNA, allowing cells to mount a canonical antiviral RNA interference response. The SF1 helicase MOV10, presented by Stephanie Ceman (University of Illinois), controls gastrulation and development of the central nervous system by binding G-rich structures and unwinding RNA in an ATP-dependent manner. Bret Pearson (Oregon Health and Science University) showed that DDX56 regulates stemness by localizing to the nucleolus in normal and cancer stem cells, regulating rRNA expression and nucleolar integrity ${ }^{9}$. In summary, RHs impart translational control and mediate the efficiency of protein synthesis, confer RNA chaperone and RNA localization functions, thus affecting cell growth and development. The discovery of antiviral Dicer, a new member of antiviral effector helicases, underscores the broad functions of RHs including their role in infection and immunity.

\section{Triggering innate immunity and the immune response against RNA viruses} The RIG-1-like receptors (RLRs) are a subfamily of RHs that have major roles as pathogen recognition receptors and initiators of innate immune activation. RLRs are increasingly implicated in immune programming in which they facilitate innate-to-adaptive immunity crosstalk. Curt Horvath (Northwestern University) reviewed RLR biology, including retinoic acid-inducible gene I (RIG-1), laboratory of genetics and physiology-2 (LGP2), and melanoma differentiation-associated gene-5 (MDA5). RLRs serve as pathogen recognition receptors by recognizing and binding to non-self pathogen associated molecular patterns (PAMPs) within viral RNA, inducing and regulating antiviral innate immune defense and linking innate and adaptive immunity ${ }^{10}$. The immune response to virus infection typically initiates with innate immunity in which during RNA virus infection the RLRs signal innate immune activation. RLR recognition of viral RNA via binding to PAMP motifs within viral RNA serves to initiate the immune response to infection. Joseph Marcotrigiano (National Institutes of Health) discussed the structural features of RIG-1 with an emphasis on viral PAMP RNA binding at the C-terminal domain (also known as the repressor domain), which triggers ATP hydrolysis and a 


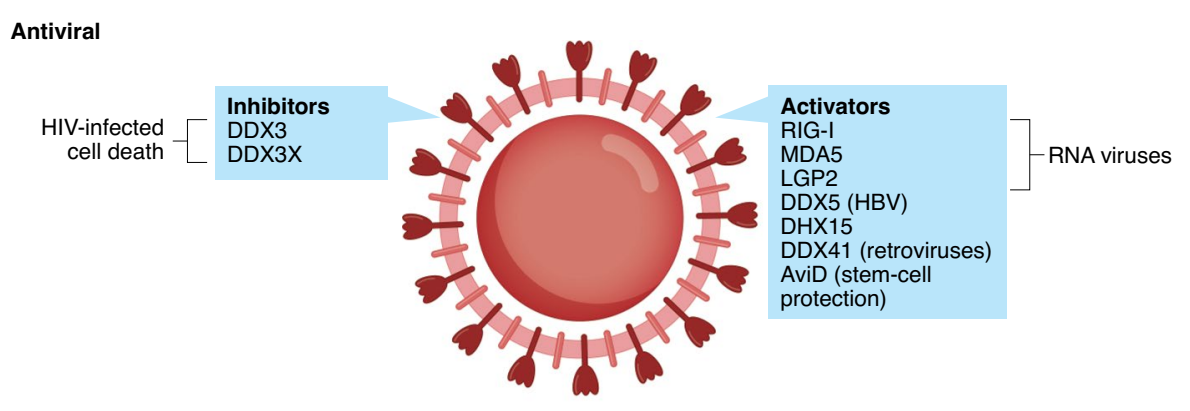

Tumor supression

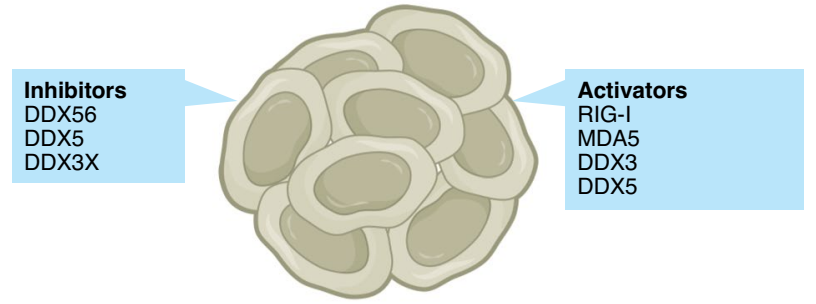

Autoinflammatory disease

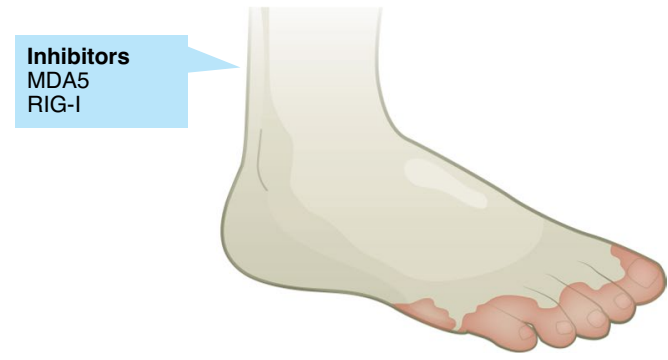

Fig. 2 | Immuno-therapeutic considerations of RHs. Top, RH inhibitors (left) or activators (right) regulate antiviral actions of specific helicases. Middle, $\mathrm{RHs}$ are linked to the suppression of specific tumor types in which $\mathrm{RH}$ inhibition (left) or activation (right) could cause tumor cell death. Bottom, aberrant activation and signaling by RIG-I and MDA5 leading to constitutive type 1 IFN production and actions are linked to a class of autoimmune conditions known as interferonopathies in which specific $\mathrm{RH}$ inhibition could ameliorate disease. AviD; antiviral Dicer; HBV, hepatitis B virus.

conformational change in RIG-1 to the signaling-on state. Although much is known about the structure-function of RIG-1, less is known of the structural properties of MDA5 regulation. Michael Gale, Jr (University of Washington) showed that SARS-CoV-2 infection in lung epithelial cells is sensed by MDA5, after its recognition and binding to specific viral RNA PAMP products. Greg Towers (University College London) further described that RLR sensing of SARS-CoV-2 promotes activation of macrophages and inflammatory actions. In particular, the Alpha (B.1.1.7) SARS-CoV-2 variant more effectively suppresses innate immune responses in airway epithelial cells by expressing high amounts of ORF9B and ORF6, which are innate immune antagonists that suppress downstream RLR signaling actions. These properties of SARS-CoV-2 reflect strategies in general across virus genera to target and suppress RLR function to avoid innate immunity, thus facilitating viral replication and spread. RLR suppression by a broad range of RNA viruses is linked to viral pathogenesis and disease. Sun Hur (Harvard Medical School) then highlighted the diverse bivalent recognition of tripartite motif (TRIM) ubiquitin ligases (RIPLET and TRIM65) by RLRs. She showed how RIPLET recognizes active, filamentous forms of RIG-1, whereas TRIM65 recognizes filamentous MDA $5^{11}$. Notably, RLR interactions with RIPLET or TRIM proteins are known to be targeted and dysregulated by pathogenic viruses. Moreover, DHX15 is another RH involved in immune activation, and was shown by Zhiqiang Zhang (Weill Cornell Medical College) to be essential for sensing rotavirus in intestinal epithelial cells, leading to the production of interferon (IFN) and IL-18. Studies presented in this session demonstrate the crucial roles of RHs and their cofactors in initiating the immune response to virus infection, showing that targeting and disruption of these processes is a hallmark feature of pathogenic RNA viruses.

\section{DEAD-H-box RHs in inflammation and immune programming}

Beyond activation of the innate immune system, RLRs and other RHs have important roles in immune programming to direct or 'polarize' the immune response toward specific effector actions. Mehul Suthar (Emory University) described how LGP2 and MAVS (the RLR signaling adaptor) regulate activation of T cells through different mechanisms - LGP2 is an RH that supports antigen-induced $\mathrm{T}$ cell expansion whereas MAVS exerts a metabolic role by integrating signals from $\mathrm{T}$ cell receptors and RLRs to program the T cells toward an inflammatory or effector antiviral phenotype. Reflecting this expanding role in immune activation and immune programming, monogenic disorders of RLRs present dire clinical outcomes. As discussed by Tracy Briggs (University of Manchester), heterozygous pathogenic mutation in IFIH1 (which encodes MDA5) is linked to skin pathologies, neurological disorders and premature tooth loss. Increased ISG expression in these disorders indicates their linkage with constitutive type I IFN signaling. MDA5/ IFN-linked disorders or 'interferonopathies' also include Aicardi-Goutières and Singleton-Merten syndromes ${ }^{12}$. Marisa Gariglio (University of Piemonte Orientale) described how human papillomavirus E6 and E7 oncoproteins evade host innate immunity by depletion of several innate immune effector RHs. Therefore, RLRs and other RHs impart immune signaling and immune programming actions that when dysregulated impose severe autoinflammatory disorders.

\section{DEAD/DEAH-box RHs in therapeutics and vaccination}

As enzymes, and owing to their broad functions in biological processes, DEAD/ DEAH-box RHs present attractive therapeutic targets to consider in strategies aimed at mitigating disease. For example, Cecil Han (Georgetown University) discussed how DDX3X knockdown in cancer cells increased type I IFN production, STAT activation and ISG expression via cytosolic accumulation of endogenous double-stranded RNAs (dsRNAs). She suggested that targeting DDX3X triggers antitumor immunity via the same mechanism and might serve as a therapeutic liability in cancer cells. Shringar Rao (Erasmus University Medical Center) described how DDX3X inhibitors can 
induce cell death and serve as a therapeutic agent against latent $\mathrm{HIV}$-infected cells; thus, approaches to inhibit DDX3X can be considered in HIV cure strategies. Moreover, RIG-1 signaling, as presented by Hendrik Poeck (Technical University of Munich), is crucial in supporting and enhancing immune checkpoint blockade-targeted tumor therapy, underscoring an emerging therapeutic application for RLR agonists as anti-tumor immune adjuvants. Simon Rothenfusser (Munich University Hospital) then focused on synthetic strategies for RIG-1 activation to improve immunotherapies for cancer. In a mouse model of acute myeloid leukemia, 5'triphosphate RNA induced RIG-1 signaling and reduced tumor burden by enhancing tumor-specific $\mathrm{T}$ cell responses in synergy with immune checkpoint blockade treatment. Lastly, Dahai Luo (Nanyang Technological University) reported that synthesized immune-modulatory RNAs can regulate RIG-I-mediated antiviral immune responses for therapy and vaccination. Studies of immune-modulatory RNAs showed impressive anti-tumor activity via enhancement of tumor-specific T cell responses in mouse models of melanoma. This session showed that targeting RIG-I alone and in combination with immune checkpoint blockade such as anti-PD-1 antibodies can offer powerful anti-cancer therapeutic potential and immune enhancement against virus infection and cancer. Moreover, mitigating RH expression, such as by the DDX3X helicase, could serve as an effective strategy to suppress certain types of solid tumor. Synthetic biology to produce and evaluate $\mathrm{RH}$ agonists and inhibitors, including RLR-specific RNA ligands and small molecule compounds, represents an exciting area of $\mathrm{RH}$ research for controlling biological processes of disease (Fig. 2).

\section{Implications and directions}

The workshop concluded with a discussion of RH functions. Considering that specific RNAs are compartmentalized in the cells ${ }^{13}$ it remains to be determined how $\mathrm{RH}$ functions link with specific RNA location, recognition, RNA secondary structure and binding. Although the DEAD/DEAH-box $\mathrm{RH}$ family is large (Fig. 1), only a few RHs were discussed in this workshop (Table 1). Future directions should include expanding research across all members of the DEAD/DEAH-box RH family. Defining the spectrum of substrate RNAs that are targeted and bound by specific RHs across tissues and under different conditions of stress, infection, cancer and autoimmunity is a perceived priority. Similarly, defining $\mathrm{RH}$-interacting proteins will continue to expand our understanding of $\mathrm{RH}$ regulation and function.

Ourania Andrisani', Qian Liu², Patricia Kehn'2, Wolfgang W. Leitner², Kyung Moon ${ }^{3}$, Nancy Vazquez-Maldonado ${ }^{2}$, lan Fingerman ${ }^{4}$ and Michael Gale Jr(D) ${ }^{5 凶}$ ${ }^{1}$ Department of Basic Medical Sciences and Purdue Center for Cancer Research, Purdue University, West Lafayette, IN, USA. ${ }^{2}$ Division of Allergy, Immunology, and Transplantation, National Institute of Allergy and Infectious Diseases, Rockville, MD, USA. ${ }^{3}$ Division of Blood Diseases and Resources, National Heart, Lung, and Blood Institute, Bethesda, MD, USA. ${ }^{4}$ Division of Cancer Biology, National Cancer Institute, Rockville, MD, USA. ${ }^{5}$ Center for Innate Immunity and Immune Disease, Department of Immunology, University of Washington, Seattle, WA, USA.

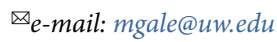

Published online: 22 February 2022

https://doi.org/10.1038/s41590-022-01149-7

\section{References}

1. Bourgeois, C. F., Mortreux, F. \& Auboeuf, D. Nat. Rev. Mol. Cell Biol. 17, 426-438 (2016).

2. Rocak, S. \& Linder, P. Nat. Rev. Mol. Cell Biol. 5, 232-241 (2004).

3. Chow, K. T., Gale, M. Jr \& Loo, Y. M. Annu. Rev. Immunol. 36 667-694 (2018).

4. Cargill, M., Venkataraman, R. \& Lee, S. Genes 12, 1471 (2021)

5. Patmore, D. M. et al. Dev. Cell 54, 455-470.e455 (2020).

6. Heerma van Voss, M. R., van Diest, P. J. \& Raman, V. Biochim Biophys Acta Rev. Cancer 1868, 510-520 (2017).

7. Zhang, H. et al. Hepatology 64, 1033-1048 (2016).

8. Weinreb, J. T. et al. Dev. Cell 56, 627-640.e625 (2021).

9. Pryszlak, M. et al. Cell Rep. 34, 108903 (2021).

10. Parisien, J. P. et al. EMBO Rep. 19, e45176 (2018).

11. Kato, K. et al. Mol. Cell 81, 599-613.e598 (2021).

12. Rice, G. I. et al. Human Mut. 41, 837-849 (2020)

13. Quinodoz, S. A. et al. Cell 184, 5775-5790.e5730 (2021).

Acknowledgements

Supported by NIH grants DK044533-23 and CA02316838S1 (O.A.) and AI143265, AI100625 and AI145359 (M.G.).

Author contributions

O.A. and M.G. served as co-organizers of this workshop, and wrote and edited portions of this report. O.A. designed Fig. 1 and Table 1. M.G. designed Fig. 2 and edited Table 1. Q.L., P.K., W.W.L., K.M., M.V.-N. and I.F. wrote portions of this report.

Competing interests

The authors declare no competing interests. 